NORDITA-2007-14

\title{
The strong coupling limit of the scaling function from the quantum string Bethe Ansatz
}

\author{
P.Y. Casteill ${ }^{a}$ and C. Kristjansen ${ }^{b}$ \\ ${ }^{a}$ The Niels Bohr Institute, Copenhagen University \\ Blegdamsvej 17, DK-2100 Copenhagen Ø, Denmark. \\ ${ }^{b}$ The Niels Bohr Institute and NORDITA, Copenhagen University \\ Blegdamsvej 17, DK-2100 Copenhagen Ø, Denmark.
}

\begin{abstract}
Using the quantum string Bethe ansatz we derive the one-loop energy of a folded string rotating with angular momenta $(S, J)$ in $A d S_{3} \times S^{1} \subset A d S_{5} \times S^{5}$ in the limit $1 \ll J \ll S, z=\sqrt{\lambda} \log (S / J) /(\pi J)$ fixed. The one-loop energy is a sum of two contributions, one originating from the Hernandez-Lopez phase and another one being due to spin chain finite size effects. We find a result which at the functional level exactly matches the result of a string theory computation. Expanding the result for large $z$ we obtain the strong coupling limit of the scaling function for low twist, high spin operators of the $S L(2)$ sector of $\mathcal{N}=4$ SYM. In particular we recover the famous $-\frac{3 \log (2)}{\pi}$. Its appearance is a result of non-trivial cancellations between the finite size effects and the Hernandez-Lopez correction.
\end{abstract}

PACS: 11.15.-q, 11.15.Me, 11.25.Tq

Keywords: cusp anomalous dimension, scaling function, strong coupling expansion, Bethe equations, AdS/CFT corrrespondence

arXiv:0705.0890 [hep-th] 


\section{Introduction}

Due to recent years discovery of integrable models underlying the spectral problems of both $\mathcal{N}=4 \mathrm{SYM}$ [1] and type IIB string theory on $A d S_{5} \times S^{5}$ [2] the spectral part of the AdS/CFT conjecture [3] can now be stated in a very pointed manner. Namely, the conjecture simply says that the $S$-matrix of the respective integrable models must agree [4]. Furthermore, the common symmetry group of the two theories constrains the S-matrix up to a phase factor [5]. The formulation of the conjecture can thus be further sharpened to the statement that the phase factors of respectively $\mathcal{N}=4 \mathrm{SYM}$ and type IIB string theory on $A d S_{5} \times S^{5}$ should be identical.

Based on educated guessing, phase factors for both $\mathcal{N}=4$ SYM and type IIB string theory on $A d S_{5} \times S^{5}$ have been put forward. In accordance with the strong-weak coupling nature of the AdS/CFT correspondence the gauge theory phase factor [6] is given as an infinite series in the 't Hooft coupling constant $\lambda$ whereas the string theory phase factor $[7]$ is given as an asymptotic expansion in $\frac{1}{\sqrt{\lambda}}$. There exist arguments that the string theory asymptotic expansion for large $\lambda$ can originate from the same function as defined by the gauge theory perturbative expansion which has a finite radius of convergence [6]. However, both phase factors are rather involved functions and it would be reassuring to see an example of a simple observable which can be extrapolated smoothly from weak to strong coupling.

A candidate for such an observable is the universal scaling function or cusp anomalous dimension, $f(g)$ where $g^{2}=\frac{\lambda}{8 \pi^{2}}$. It is related to the anomalous dimension of low twist operators of $\mathcal{N}=4 \mathrm{SYM}$ of the type

$$
\mathcal{O}=\operatorname{Tr}\left(D^{S} Z^{J}+\ldots\right)
$$

Here $D$ is a light cone derivative, $Z$ is a complex scalar, $S$ is the space-time spin and $J$ is denoted as the twist. For leading twist, i.e. $J=2$, it is well-known that the anomalous dimension $\Delta$ of such an operator for large values of the spin grows logarithmically with the spin

$$
\Delta-S=f(g) \log (S), \quad S \rightarrow \infty,
$$

where $f(g)$ can be expanded perturbatively in $g$. The scaling function has the appealing feature that, as opposed to other observables one could think of, it depends only on one parameter $g$. For instance, it is not polluted by any additional $J$-dependence. The function $f(g)$ has been determined by solid field theory calculations up to and including four-loop order [8]. Furthermore, starting from the asymptotic gauge theory Bethe equations [9], inserting the conjectured gauge theory phase factor [6] and taking a large- $S$ limit it has been possible to derive an equation which determines $f(g)$ to all orders in $g$ [10]. This equation, known as the BES equation, correctly reproduces the known first four orders in $g^{2}$. Its derivation, however, relies on the assumption that the scaling function is the same for all operators with a finite value of the twist and that at the same time it is permitted to take $J$ sufficiently large so that the asymptotic Bethe equations are correct.

On the string theory side a low twist, high spin operator corresponds to a folded string rotating with angular momentum $S$ on $A d S_{3} \subset A d S_{5} \times S^{5}$ [11]. The energy of such a 
string has an expansion for large $\lambda$ which reads

$$
E=\left(\frac{\sqrt{\lambda}}{\pi}-\frac{3 \log (2)}{\pi}+\mathcal{O}\left(\frac{1}{\sqrt{\lambda}}\right)\right) \log S, \quad S \rightarrow \infty
$$

Here the first term follows from semi-classical analysis [11] and the second one from a oneloop computation [12]. Deriving this result from the Bethe equations would yield a very comforting confirmation of both the integrability approach as well as of the AdS/CFT conjecture itself. However, the strong coupling analysis of the BES equation has proved hard. For the moment only the leading semi-classical contribution has been derived from the BES equation by analytic means [13-15]. By numerical analysis of the equation both the leading $[16,17]$ and the next to leading order term [16] can be reproduced with high accuracy. Furthermore, it is possible to predict numerically the next term in the expansion which would result from a string theory two-loop computation [16]. In the present paper we shall consider an alternative way of obtaining an expansion à la (3) by Bethe equation techniques.

An operator of the type (1) for which $J$ is not finite has a string theory dual which in addition to the angular momentum, $S$ on $A d S_{3}$ carries an angular momentum $J$ on $S^{1} \subset S^{5}$. For such a string, considering the situation

$$
1 \ll J \ll S, \quad z \equiv \frac{\sqrt{\lambda}}{\pi J} \log \left(\frac{S}{J}\right), \quad \text { fixed },
$$

one finds that the semi-classical [18] as well as the one loop energy [19] can be written down in a closed form as a function of $z$. Furthermore, the formula obtained for the string energy interpolates smoothly between small and large values of $z$ and the large- $z$ expansion looks as (3) just with the replacement $\log S \rightarrow \log \left(\frac{S}{J}\right)$. We shall discuss this string solution and the various expansions of its energy in more detail shortly. Subsequently, we will show how to reproduce the precise functional dependence of the string energy on $z$ from the string Bethe equations. In particular, we will derive by analytic means the celebrated $-\frac{3 \log (2)}{\pi}$. Our starting point will be the asymptotic Bethe equations, whose application is now more justified since we take $J \gg 1$, supplemented with the conjectured string phase factor. The classical string energy as a function of $z$ is obtained almost immediately by considering only the AFS phase [20] whereas the one-loop energy requires more work. For one we have to take into account the HL-correction [21] to the phase and secondly we have to consider spin-chain finite size effects [22]. As we shall see we are able to determine the contribution from each of these effects exactly as a function of $z$. The $-\frac{3 \log (2)}{\pi}$ results from a non-trivial cancellation between the two types of terms as $z \rightarrow \infty$.

We start in section 2 by recalling from reference [19] the description of the folded string rotating on $A d S_{3} \times S^{1} \subset A d S_{5} \times S^{5}$ in the limit given by eqn. (4). In section 3 we write down the relevant string Bethe equations and perform the necessary expansions. After that, in sections 4 and 5, we extract from these respectively the semi-classical and the one-loop energy. Finally, section [6] contains our conclusion. 


\section{The folded string spinning on $A d S_{3} \times S^{1}$}

A folded string living in $A d S_{5} \times S^{5}$ and carrying large angular momenta $S$ and $J$ on respectively $A d S_{3}$ and $S^{1}$ is a system which has successfully been studied in the semiclassical approximation. Hence, its classical energy was determined in [12]. The expression for the energy simplifies considerably in the limit given in eqn. (4), i.e. [18]

$$
1 \ll J \ll S, \quad z \equiv \frac{\sqrt{\lambda}}{\pi J} \log \left(\frac{S}{J}\right) \quad \text { fixed }
$$

One finds

$$
E_{0}=S+J \sqrt{1+z^{2}}
$$

Expanding for large $z$ we get

$$
E_{0}(z \gg 1)=S+\frac{\sqrt{\lambda}}{\pi} \log \left(\frac{S}{J}\right)+\ldots
$$

Here we notice the leading strong coupling term announced earlier, cf. eqn. (3). For $z \ll 1$ one recovers what is known as the fast spinning string solution [23]

$$
E_{0}(z \ll 1)=S+J+\frac{\lambda}{2 \pi^{2} J} \log ^{2}\left(\frac{S}{J}\right)-\frac{\lambda^{2}}{8 \pi^{4} J^{3}} \log ^{4}\left(\frac{S}{J}\right)+\frac{\lambda^{3}}{16 \pi^{6} J^{5}} \log ^{6}\left(\frac{S}{J}\right)+\ldots
$$

The first logarithmic term was reproduced in the Bethe ansatz approach in [24] and the second one is contained in the work in [18]. Later, we shall show that when the limit (5) is imposed from the beginning in the all Loop Bethe ansatz, the exact square root formula immediately appears. Recently, an expression for the one-loop contribution to the energy in the same limit was derived [19]. The result reads

$$
\begin{aligned}
E_{1}=\frac{J}{\sqrt{\lambda}} \frac{1}{\sqrt{1+z^{2}}}\left\{z \sqrt{1+z^{2}}-\left(1+2 z^{2}\right) \log \left[z+\sqrt{1+z^{2}}\right]\right. \\
\left.-z^{2}+2\left(1+z^{2}\right) \log \left(1+z^{2}\right)-\left(1+2 z^{2}\right) \log \left[\sqrt{1+2 z^{2}}\right]\right\}
\end{aligned}
$$

It is obtained under the further assumption that

$$
\frac{J}{\sqrt{\lambda}} \sqrt{1+z^{2}} \gg 1
$$

Expanding (9) for small $z$, we get for the fast spinning case

$$
E_{1}(z \ll 1)=-\frac{4 \lambda}{3 \pi^{3} J^{2}} \log ^{3}\left(\frac{S}{J}\right)+\frac{4 \lambda^{2}}{5 \pi^{5} J^{4}} \log ^{5}\left(\frac{S}{J}\right)+\frac{\lambda^{5 / 2}}{3 \pi^{6} J^{5}} \log ^{6}\left(\frac{S}{J}\right)+\ldots
$$

Taking in stead $z$ to be large, one finds

$$
E_{1}(z \gg 1)=-\frac{3 \log (2)}{\pi} \log \left(\frac{S}{J}\right)+\ldots
$$


Here we recognize the famous $-\frac{3 \log (2)}{\pi}$ coefficient from the large- $\lambda$ expansion (3)). As we shall explain in the next section, from the Bethe equation perspective it is natural to separate $E_{1}$ into a part which is analytic in $\lambda$ for small $\lambda$ and one which is not. Terms which are analytic, respectively non-analytic, in $\lambda$ originate from terms which are odd, respectively even, in $z$. (The even terms constitute the first line in eqn. (9) and the odd ones the second line.) Explicitly, we have

$$
\begin{aligned}
\left(E_{1}\right)_{\text {string }}^{\text {analytic }}= & \frac{J}{\sqrt{\lambda}}\left(z-\frac{1+2 z^{2}}{\sqrt{1+z^{2}}} \log \left[z+\sqrt{1+z^{2}}\right]\right) \\
= & -\frac{4 \lambda \log ^{3}\left(\frac{S}{J}\right)}{\pi^{3} J^{2}}\left(\frac{1}{3}-\frac{1}{5} z^{2}+\frac{16}{105} z^{4}+\ldots\right), \\
\left(E_{1}\right)_{\text {string }}^{\text {non-analytic }}= & \frac{J}{\sqrt{\lambda}} \frac{1}{\sqrt{1+z^{2}}}\left(-z^{2}+2\left(1+z^{2}\right) \log \left(1+z^{2}\right)\right. \\
& \left.-\left(1+2 z^{2}\right) \log \left[\sqrt{1+2 z^{2}}\right]\right) \\
= & \frac{\lambda^{5 / 2} \log ^{6}\left(\frac{S}{J}\right)}{\pi^{6} J^{5}}\left(\frac{1}{3}-\frac{2}{3} z^{2}+\frac{43}{40} z^{4}+\ldots\right) .
\end{aligned}
$$

The first term in the expansion (14) of the analytic part was recovered using the oneloop Bethe ansatz in [18]. Below we shall recover the exact functional expressions (13) and (15). It is an important point to notice that the appearance of the $-\frac{3 \log (2)}{\pi}$ term for large $z$ is due to non-trivial cancellations between the analytic and the non-analytic part. More precisely, we have

$$
\begin{aligned}
\left(E_{1}\right)_{\text {string }}^{\text {analytic }} & \sim\left(\frac{-2 \log (z)+1-2 \log (2)}{\pi}\right) \log \left(\frac{S}{J}\right) \quad \text { as } \quad z \rightarrow \infty, \\
\left(E_{1}\right)_{\text {string }}^{\text {non-analytic }} & \sim\left(\frac{2 \log (z)-1-\log (2)}{\pi}\right) \log \left(\frac{S}{J}\right) \quad \text { as } \quad z \rightarrow \infty .
\end{aligned}
$$

\section{The string Bethe equations}

The spectrum of strings moving on $A d S_{3} \times S^{1} \subset A d S_{5} \times S^{5}$ is encoded in the Bethe equations of a generalized $\mathfrak{s l}(2)$ spin chain, i.e.

$$
\left(\frac{x_{k}^{+}}{x_{k}^{-}}\right)^{J}=\prod_{j \neq k}^{S}\left(\frac{x_{k}^{-}-x_{j}^{+}}{x_{k}^{+}-x_{j}^{-}}\right) \frac{1-g^{2} / 2 x_{k}^{+} x_{j}^{-}}{1-g^{2} / 2 x_{j}^{+} x_{k}^{-}} \sigma^{2}\left(x_{k}, x_{j}\right),
$$

Here $S$ and $J$ are representation labels associated with the angular momentum of the string on respectively $A d S_{3}$ and $S^{1}$ and $g$ is the inverse string tension

$$
g^{2}=\frac{\lambda}{8 \pi^{2}} \sim \frac{1}{\alpha^{2}} .
$$

The indices $j, k$ label elementary excitations and the $x^{ \pm}$variables are related to the momenta carried by these excitations via

$$
\exp (\mathrm{i} p)=\frac{x^{+}}{x^{-}}
$$


Furthermore, the quantity $\sigma\left(x_{k}, x_{j}\right)$ is the phase factor, restricted by symmetry arguments to be of the form [25]

$$
\begin{aligned}
\sigma\left(x_{k}, x_{j}\right) & =e^{\mathrm{i} \theta\left(x_{k}, x_{j}\right)} \\
\theta\left(x_{k}, x_{j}\right) & =\sum_{r=2}^{\infty} \sum_{s=r+1}^{\infty}\left(\frac{g^{2}}{2}\right)^{(r+s-1) / 2} c_{r, s}(g)\left[q_{r}\left(x_{k}\right) q_{s}\left(x_{j}\right)-q_{r}\left(x_{j}\right) q_{s}\left(x_{k}\right)\right],
\end{aligned}
$$

where the charges $q_{r}(x)$ (with $r \geq 2$ ) are defined by

$$
q_{r}\left(x_{k}\right)=\frac{\mathrm{i}}{r-1}\left(\frac{1}{\left(x_{k}^{+}\right)^{r-1}}-\frac{1}{\left(x_{k}^{-}\right)^{r-1}}\right), \quad Q_{r}=\sum_{k} q_{r}\left(x_{k}\right) .
$$

In the string theory description, the $c_{r, s}$ coefficients are expected to have an expansion in $\alpha^{\prime} \sim \frac{1}{\sqrt{\lambda}}$

$$
c_{r, s}(\lambda)=c_{r, s}^{(0)}+\frac{1}{\sqrt{\lambda}} c_{r, s}^{(1)}+\frac{1}{\lambda} c_{r, s}^{(2)}+\ldots,
$$

and the string phase factor conjecture [7] accordingly involves an explicit conjecture for the $c_{r, s}^{(i)}$. The first two terms can be determined by comparing to conventional string theory computations and read $[20,21]$

$$
\begin{aligned}
c_{r, s}^{(0)} & =\delta_{s, r+1}, \\
c_{r, s}^{(1)} & =-4\left(1-(-1)^{r+s}\right) \frac{(r-1)(s-1)}{(s+r-2)(s-r)} .
\end{aligned}
$$

In order to describe proper string states the Bethe equations must be supplemented by the level matching or momentum condition

$$
\prod_{k=1}^{S}\left(\frac{x_{k}^{+}}{x_{k}^{-}}\right)=1
$$

and finally the string energy is then obtained as

$$
E=\frac{\lambda}{8 \pi^{2}} Q_{2}
$$

Now, our aim is to determine the classical and the one-loop energy of a certain string configuration in the limit given by eqn. (5). For that purpose we need to expand the phase factor to two leading orders in $\alpha^{\prime} \sim \frac{1}{\sqrt{\lambda}}$., i.e. to take into account $c_{r, s}^{(0)}$ and $c_{r, s}^{(1)}$ above. Correspondingly, we have to expand all terms to two leading orders in $\frac{1}{J}$. In order to perform the large- $J$ expansion we need to express the $x$-variables via a rapidity variable $u$ in the following way

$$
\begin{aligned}
x^{ \pm} & =x(u \pm \mathrm{i} / 2), \\
x(u) & =\frac{u}{2}+\frac{u}{2} \sqrt{1-\frac{2 g^{2}}{u^{2}}}, \\
u(x) & =x+\frac{g^{2}}{2 x} .
\end{aligned}
$$


We then rescale the variables $x=x(u)$ and $g^{2}$ in the following way

$$
\begin{aligned}
x=x(u) & \rightarrow J x, \\
g^{2} & \rightarrow g^{2} J^{2} .
\end{aligned}
$$

Taking the logarithm of the Bethe equations and expanding to the relevant order in $J$ and $\lambda$ we obtain

$$
\begin{aligned}
& -\frac{1}{x_{k}\left(1-g^{2} /\left(2 x_{k}^{2}\right)\right)}+2 \pi m_{k}= \\
& \quad \frac{2}{J} \sum_{j \neq k}^{S} \frac{1}{\left(x_{k}-x_{j}\right)\left(1-g^{2} /\left(2 x_{j}^{2}\right)\right)}-\frac{2}{J} \frac{g^{2}}{2 x_{k}^{2}} \frac{1}{1-g^{2} /\left(2 x_{k}^{2}\right)} \sum_{j \neq k}^{S} \frac{1}{1-g^{2} /\left(2 x_{j}^{2}\right)} \frac{1}{x_{j}} \\
& \quad+\frac{1}{J} \text { Anomaly }\left(x_{k}\right)+\frac{1}{J} \operatorname{Non}-\operatorname{analytic}\left(x_{k}\right),
\end{aligned}
$$

where $m_{k}$ is a mode number coming from the ambiguity of the logarithm. The two first lines constitute the classical Bethe equations and the last line contains the one-loop correction. The one-loop correction consists of two terms. The term Anomaly $\left(x_{k}\right)$ is a spin chain finite size effect. It arises due to the fact that the naive expansion of the logarithm becomes invalid when $x_{j}-x_{k} \sim \mathcal{O}(1 / J)$ [22]. This term is analytic in $\lambda$. As indicated by the notation, the other one-loop term is non-analytic in $\lambda$. It is the part of $\theta\left(x_{j}, x_{k}\right)$ which originates from the $\frac{1}{\sqrt{\lambda}}$ term in eqn. (24), i.e. the Hernandez-Lopez phase [21]. Notice that the leading part of $\theta\left(x_{j}, x_{k}\right)$, i.e. the AFS phase [20], contributes already at the classical level. Now we make the assumption about the distribution of Bethe roots that is known to lead to the folded string solution [24], namely we assume that the roots lie in two intervals $[-b,-a]$ and $[a, b]$ on the real axis and are symmetrically distributed around zero. This means that the second term on the right hand side of eqn. (34) vanishes 1 Furthermore, we assign the mode number $-n$ to roots lying in the right interval and mode number $+n$ to roots lying in the left interval. Finally, we introduce a resolvent corresponding to the roots lying in the right interval

$$
G(x)=\frac{1}{J} \sum_{j=1}^{S / 2} \frac{1}{x-x_{j}} \frac{1}{1-g^{2} /\left(2 x_{j}^{2}\right)} \equiv \int_{a}^{b} \mathrm{~d} y \frac{\rho(y)}{x-y},
$$

and we assume that $G(x)$ has a well-defined expansion in $\frac{1}{J} \sim \frac{1}{\sqrt{\lambda} \log (S / J)}$, i.e.

$$
G(x)=G_{0}(x)+\frac{1}{J} G_{1}(x)+\ldots,
$$

where each $G_{i}(x)$ is analytic in the complex plane except for a cut $[a, b]$. Accordingly, the density $\rho(x)$ needs to have a well-defined $\frac{1}{J}$ expansion

$$
\rho(x)=\rho_{0}(x)+\frac{1}{J} \rho_{1}(x)+\ldots
$$

\footnotetext{
${ }^{1}$ The fact that the sum in this term does not include the root at $j=k$ is an $1 / J$ effect which can be ignored as the term does not have any accompanying factors of $\log \left(\frac{S}{J}\right)$.
} 
with each term in the expansion having support on the interval $[a, b]$. The normalization condition for $\rho(x)$ reads

$$
\int_{a}^{b} \mathrm{~d} y \rho(y)\left\{1-\frac{g^{2}}{2 y^{2}}\right\}=\frac{S}{2 J} \equiv \frac{\alpha}{2}
$$

and the string energy, $E$, is encoded in $\rho(y)$ in the following way

$$
\int_{a}^{b} \mathrm{~d} y \rho(y)=\frac{S}{2 J}+\frac{E-S-J}{4 J} .
$$

If we write

$$
G(x)=G_{+}(x)+x G_{-}(x), \quad \text { where } \quad G_{ \pm}(x)=G_{ \pm}(-x),
$$

we have

$$
E=J+S+2 J g^{2} \int \mathrm{d} x \frac{\rho(x)}{x^{2}}=J+S-2 J g^{2} G_{-}(0) .
$$

Using the resolvent we can write the Bethe equation in the classical limit as

$$
G_{0}(x+\mathrm{i} 0)+G_{0}(x-\mathrm{i} 0)-2 G_{0}(-x)=-\frac{1 / x}{1-g^{2} /\left(2 x^{2}\right)}+2 \pi n, \quad x \in[a, b] .
$$

This equation (42) is nothing but the saddle point equation of the $O(n)$ model on a random lattice for $n=-2[26]$ with the terms on the right hand side playing the role of the derivative of the potential. Its solution with the given boundary conditions can be written in various ways $[27,28]$. Here we shall use the formulation of $[28]$ where the solution is given in closed form for any potential using contour integrals. In order to find the one-loop correction to the string energy we have to take into account also the two last terms in eqn. (34). These terms can, at the order considered, be expressed in terms of the leading order density as follows

$$
\operatorname{Anomaly}(x)=-\frac{1}{1-g^{2} /\left(2 x^{2}\right)}\left(\pi \rho_{0}^{\prime}(x)\right)\left(\operatorname{coth}\left(\pi \rho_{0}(x)\right)-\frac{1}{\pi \rho_{0}(x)}\right),
$$

and

$$
\operatorname{Non-analytic}(x)=\frac{1}{\pi} \frac{x^{2}}{x^{2}-g^{2} / 2} \int_{a}^{b} \mathrm{~d} y \rho_{0}(y)[\Delta \phi(x, y)+\Delta \phi(x,-y)]
$$

where

$$
\Delta \phi(x, y)=\frac{1}{2} \sum_{r=2}^{\infty} \sum_{m=0}^{\infty} c_{r, 2 m+r+1}^{(1)}\left(\frac{g}{\sqrt{2}}\right)^{2 m+2 r-1}\left(\frac{1}{x^{r} y^{2 m+r+1}}-\frac{1}{x^{2 m+r+1} y^{r}}\right) .
$$

Notice that we have taken into account the fact that the full set of Bethe roots is distributed symmetrically around zero by forming the combination $[\Delta \phi(x, y)+\Delta \phi(x,-y)]$. 


\section{The semi-classical string energy}

As mentioned above the leading order equation (42) is nothing but the saddle point equation of the $O(n)$ model on a random lattice for $n=-2$ and its solution can conveniently be written down using contour integrals [28]

$$
\begin{aligned}
G_{0-}(x) & =\frac{1}{2} \oint_{\mathcal{C}_{+}} \frac{\mathrm{d} y}{2 \pi \mathrm{i}} \frac{V_{0}^{\prime}(y)}{x^{2}-y^{2}}\left\{\frac{\left(x^{2}-a^{2}\right)^{1 / 2}\left(x^{2}-b^{2}\right)^{1 / 2}}{\left(y^{2}-a^{2}\right)^{1 / 2}\left(y^{2}-b^{2}\right)^{1 / 2}}\right\}, \\
G_{0+}(x) & =2 \oint_{\mathcal{C}_{+}} \frac{\mathrm{d} y}{2 \pi \mathrm{i}} G_{-}(y) \frac{y^{2}}{x^{2}-y^{2}},
\end{aligned}
$$

where the contour encircles $\mathcal{C}_{+}=[a, b]$ counterclockwise and where

$$
V_{0}^{\prime}(y)=-\frac{1 / y}{1-g^{2} /\left(2 y^{2}\right)}+2 \pi n
$$

The endpoints of $\mathcal{C}_{+}, a$ and $b$, are determined by

$$
\oint_{\mathcal{C}_{+}} \frac{\mathrm{d} y}{2 \pi \mathrm{i}} \frac{V_{0}^{\prime}(y)}{\left(y^{2}-a^{2}\right)^{1 / 2}\left(y^{2}-b^{2}\right)^{1 / 2}}=0
$$

and

$$
\oint_{\mathcal{C}_{+}} \frac{\mathrm{d} y}{2 \pi \mathrm{i}} \frac{V_{0}^{\prime}(y) y^{2}}{\left(y^{2}-a^{2}\right)^{1 / 2}\left(y^{2}-b^{2}\right)^{1 / 2}}+\frac{g^{2}}{2} \oint_{\mathcal{C}_{+}} \frac{\mathrm{d} y}{2 \pi \mathrm{i}} \frac{V_{0}^{\prime}(y) a b}{y^{2}\left(y^{2}-a^{2}\right)^{1 / 2}\left(y^{2}-b^{2}\right)^{1 / 2}}=\frac{S}{J} .
$$

The first condition expresses the fact that $G(x)$ should tend to 0 as $x$ tends to infinity, and the second condition is a rewriting of eqn. (38). We need that the Bethe roots stay away from the singularities of the potential, i.e. the points $y=0$ and $y= \pm \frac{g}{\sqrt{2}}$. This means that we must have $g^{2}<2 a^{2}$ or $2 b^{2}<g^{2}$. We choose to work with the former assumption, i.e.

$$
g^{2}<2 a^{2}
$$

as this will directly reproduce the result of [24] in the case $g=0$. Inserting the explicit expression (48) for the potential $V_{0}^{\prime}(y)$ the boundary conditions read

$$
0=\frac{2 n}{b} K\left(k^{\prime}\right)-\frac{1}{2} \frac{1}{\sqrt{\left(a^{2}-g^{2} / 2\right)\left(b^{2}-g^{2} / 2\right)}},
$$

and

$$
\begin{aligned}
\frac{S}{J}= & 2 n b E\left(k^{\prime}\right)-\frac{1}{2}-\frac{1}{2} \frac{g^{2} / 2}{\sqrt{\left(a^{2}-g^{2} / 2\right)\left(b^{2}-g^{2} / 2\right)}}+n g^{2} \frac{1}{a} E\left(k^{\prime}\right) \\
+ & \frac{1}{2}\left\{1-\frac{a b}{\sqrt{\left(a^{2}-g^{2} / 2\right)\left(b^{2}-g^{2} / 2\right)}}\right\}
\end{aligned}
$$

where $K\left(k^{\prime}\right)$ and $E\left(k^{\prime}\right)$ are standard elliptic integrals of the first and the second kind respectively, with $k^{\prime}$ being given by

$$
k=\frac{a}{b}, \quad k^{\prime}=\left(1-k^{2}\right)^{1 / 2} .
$$


Furthermore, the expression for the semi-classical string energy takes the form

$$
\begin{aligned}
E_{0}-S-J & =-a b J g^{2} \oint_{\mathcal{C}_{+}} \frac{\mathrm{d} \omega}{2 \pi \mathrm{i}} \frac{V^{\prime}(\omega)}{\omega^{2}\left(\omega^{2}-a^{2}\right)^{1 / 2}\left(\omega^{2}-b^{2}\right)^{1 / 2}} \\
& =-J g^{2}\left\{\frac{2 n}{a} E\left(k^{\prime}\right)+\frac{1}{g^{2}}\left[1-\frac{a b}{\sqrt{\left(a^{2}-g^{2} / 2\right)\left(b^{2}-g^{2} / 2\right)}}\right]\right\} .
\end{aligned}
$$

Considering only the terms of leading order in $g$ we reproduce the results of [24], namely

$$
a=\frac{1}{4 n K\left(k^{\prime}\right)}, \quad \alpha+\frac{1}{2}=\frac{E\left(k^{\prime}\right)}{2 K\left(k^{\prime}\right)} \frac{1}{k},
$$

and

$$
E_{0}-S-J=\frac{\lambda n^{2}}{2 \pi^{2} J} K\left(k^{\prime}\right)\left\{\left(1+k^{2}\right) K\left(k^{\prime}\right)-2 E\left(k^{\prime}\right)\right\} .
$$

It is obvious that by means of the equations (52), (53) and (55) one can recursively express the semi-classical energy order by order in $\lambda$. This idea has been pursued f.inst. in references $[18,29]$. Here, we shall in stead consider the limit (44)

$$
1 \ll J \ll S, \quad z=\frac{\sqrt{\lambda} n}{\pi J} \log \left(\frac{S}{J}\right) \quad \text { fixed, }
$$

where it is possible to obtain a closed expression for the all-loop energy. We immediately see that in this limit we have

$$
k \rightarrow 0, \quad a \rightarrow 0, \quad b \rightarrow \infty
$$

and from the second boundary equation (53) we find

$$
\log \left(\frac{S}{J}\right) \sim K\left(k^{\prime}\right) \sim \log \left(\frac{1}{k}\right) .
$$

Introducing the notation

$$
\hat{g}=\frac{g}{\sqrt{2} a},
$$

we notice that the first boundary boundary equation (52) can be written as

$$
a=\frac{1}{4 n \sqrt{1-\hat{g}^{2}} \sqrt{1-k^{2} \hat{g}^{2}} K\left(k^{\prime}\right)},
$$

and therefore in the limit (58) we have

$$
\hat{g}^{2}=\frac{z^{2}}{z^{2}+1}
$$

and in particular $g^{2}<2 a^{2}$. Using eqn. (62) we can express the energy as

$$
E_{0}=S+J \frac{1}{\sqrt{\left(1-\hat{g}^{2}\right)\left(1-k^{2} \hat{g}^{2}\right)}}\left[1-\hat{g}^{2} \frac{E\left(k^{\prime}\right)}{K\left(k^{\prime}\right)}\right] .
$$


From here we immediately find, in the limit given by eqn. (58),

$$
E_{0}=S+J \sqrt{1+z^{2}}
$$

which agrees exactly with the string theory result of reference [19], cf. eqn (6) $)$. From our result for $G_{0-}$ we can extract the Bethe root distribution at leading order $\rho_{0}(x)$ in terms of which the one loop correction terms are expressed. One finds

$$
\begin{aligned}
\rho_{0}(x) & =\frac{x}{i \pi}\left(G_{0-}(x-\mathrm{i} 0)-G_{0-}(x+\mathrm{i} 0)\right) \\
& =-\frac{x}{\pi}\left(x^{2}-a^{2}\right)^{1 / 2}\left(b^{2}-x^{2}\right)^{1 / 2} \oint_{\mathcal{C}_{+}} \frac{\mathrm{d} y}{2 \pi \mathrm{i}} \frac{V^{\prime}(y)}{x^{2}-y^{2}}\left\{\frac{1}{\left(y^{2}-a^{2}\right)^{1 / 2}\left(y^{2}-b^{2}\right)^{1 / 2}}\right\} \\
& =-\frac{x}{\pi}\left(x^{2}-a^{2}\right)^{1 / 2}\left(b^{2}-x^{2}\right)^{1 / 2} f_{a}^{b} \frac{\mathrm{d} y}{\pi} \frac{V^{\prime}(y)}{x^{2}-y^{2}}\left\{\frac{1}{\left(y^{2}-a^{2}\right)^{1 / 2}\left(b^{2}-y^{2}\right)^{1 / 2}}\right\} \\
& =\frac{x \sqrt{x^{2}-a^{2}}}{2 \pi b \sqrt{b^{2}-x^{2}}}\left(\frac{b}{x^{2}-\frac{g^{2}}{2}} \frac{\sqrt{b^{2}-\frac{g^{2}}{2}}}{\sqrt{a^{2}-\frac{g^{2}}{2}}}-4 n \Pi\left(1-\frac{x^{2}}{b^{2}}, k^{\prime}\right)\right)
\end{aligned}
$$

where in the last integral the principal value appears. Considering only leading order in $g$ and setting $n=1$ we recover the expression obtained in [24]. Introducing

$$
\omega=\frac{x}{a}
$$

we can also write

$$
\rho_{0}(\omega)=\frac{2 k n \omega \sqrt{\omega^{2}-1}}{\pi \sqrt{\frac{1}{k^{2}}-\omega^{2}}}\left(\frac{\frac{1}{k^{2}}-\hat{g}^{2}}{\omega^{2}-\hat{g}^{2}} K\left(k^{\prime}\right)-\Pi\left(1-k^{2} \omega^{2}, k^{\prime}\right)\right),
$$

where now the normalization condition reads

$$
\int_{a}^{b} \mathrm{~d} x \rho_{0}(x)\left\{1-\frac{g^{2}}{2 y^{2}}\right\}=a \int_{1}^{1 / k} \mathrm{~d} \omega \rho_{0}(\omega)\left\{1-\frac{\hat{g}^{2}}{\omega^{2}}\right\}=\frac{S}{2 J} .
$$

We also note the formula

$$
\begin{aligned}
& \rho_{0}^{\prime}(\omega)=\frac{2 n}{k \pi \sqrt{\frac{1}{k^{2}}-\omega^{2}} \sqrt{\omega^{2}-1}} \times \\
& \quad\left\{\left(k^{2} \hat{g}^{2}+\frac{\left(1+\left(1-2 \hat{g}^{2}\right) k^{2}\right) \hat{g}^{2}}{\hat{g}^{2}-\omega^{2}}+\frac{\left(1-\hat{g}^{2}\right)\left(1-k^{2} \hat{g}^{2}\right)\left(\hat{g}^{2}+\omega^{2}\right)}{\left(\hat{g}^{2}-\omega^{2}\right)^{2}}\right) K\left(k^{\prime}\right)-E\left(k^{\prime}\right)\right\} .
\end{aligned}
$$

Taking the limit (4), we get

$$
\begin{aligned}
\rho_{0}(\omega) & \approx \frac{n}{\pi} \frac{\sqrt{\omega^{2}-1}}{\omega}\left\{2 \log (\alpha) \sqrt{1-k^{2} \omega^{2}} \frac{\hat{g}^{2}}{\omega^{2}-\hat{g}^{2}}+\log \left[\frac{1+\sqrt{1-k^{2} \omega^{2}}}{1-\sqrt{1-k^{2} \omega^{2}}}\right]\right\} \\
& \approx \frac{2 n}{\pi} \frac{\omega \sqrt{\omega^{2}-1}}{\omega^{2}-\hat{g}^{2}} \log (\alpha),
\end{aligned}
$$

where the latter expression of course needs to be treated with some care. Furthermore,

$$
\rho_{0}^{\prime}(\omega)=\frac{2 n}{\pi} \frac{\left(1-2 \hat{g}^{2}\right) \omega^{2}+\hat{g}^{2}}{\sqrt{\omega^{2}-1}\left(\omega^{2}-\hat{g}^{2}\right)^{2}} \log (\alpha) .
$$




\section{The one-loop string energy}

Including the one-loop corrections, our Bethe equations read

$$
G(x+\mathrm{i} 0)+G(x-\mathrm{i} 0)-2 G(-x)=V_{0}^{\prime}(x)+\frac{1}{J} V_{1}^{\prime}(x),
$$

with $V_{0}^{\prime}(x)$ given by eqn. (48) and with

$$
V_{1}^{\prime}(x)=\operatorname{Anomaly}(x)+\operatorname{Non}-\operatorname{analytic}(x) .
$$

By applying the solution formula (46) to this equation and expanding everything including the interval boundaries in $\frac{1}{J}$, one derives the following formula for $G_{1-}(x)$

$$
G_{1-}(x)=\frac{1}{2} \oint_{\mathcal{C}_{+}} \frac{\mathrm{d} \omega}{2 \pi \mathrm{i}} V_{1}^{\prime}(\omega)\left(\frac{1}{x^{2}-\omega^{2}}-\frac{\frac{g^{2}}{2 a b}}{1-\frac{g^{2}}{2 a b}} \frac{1}{\omega^{2}}\right)\left\{\frac{\left(\omega^{2}-a^{2}\right)^{1 / 2}\left(\omega^{2}-b^{2}\right)^{1 / 2}}{\left(x^{2}-a^{2}\right)^{1 / 2}\left(x^{2}-b^{2}\right)^{1 / 2}}\right\},
$$

where we stress that the points $a$ and $b$ are the same as for the leading order solution. The one-loop contribution to the energy then reads, cf. eqn. (41)

$$
E_{1}=-2 g^{2} G_{1-}(0)
$$

\subsection{The spin chain finite size correction}

As explained above the spin chain finite size corrections will give us the analytic part of the one-loop string energy. This contribution is determined from (76) by inserting $\operatorname{Anomaly}(x)$ at the place of $V_{1}^{\prime}(x)$ in eqn. (75) . One gets

$$
\begin{aligned}
& \left(E_{1}\right)_{\text {Bethe }}^{\text {analytic }}= \\
& \quad-\frac{\frac{g^{2}}{a b}}{1-\frac{g^{2}}{a b}} \int_{a}^{b} \frac{\mathrm{d} x}{\pi} \frac{1}{1-\frac{g^{2}}{2 x^{2}}}\left(\pi \rho_{0}^{\prime}(x)\right)\left(\operatorname{coth}\left(\pi \rho_{0}(x)\right)-\frac{1}{\pi \rho_{0}(x)}\right) \frac{\sqrt{\left(x^{2}-a^{2}\right)\left(b^{2}-x^{2}\right)}}{x^{2}} .
\end{aligned}
$$

In the limit we are interested in, $\rho_{0}(x)$ and $\rho_{0}^{\prime}(x)$ are given by eqns. (71) and (72). In particular, since $\rho_{0}(x)$ contains the divergent factor $\log (\alpha)$ we can use the approximation $\operatorname{coth}\left(\pi \rho_{0}(x)\right)-\frac{1}{\pi \rho_{0}(x)}=1$. In this way the integral above becomes

$$
\begin{aligned}
\left(E_{1}\right)_{\text {Bethe }}^{\text {analytic }} & =-\frac{g^{2}}{a^{2}}(2 n \log (\alpha)) \int_{1}^{\infty} \frac{\mathrm{d} \omega}{\pi} \frac{\left(1-2 \hat{g}^{2}\right) \omega^{2}+\hat{g}^{2}}{\left(\omega^{2}-\hat{g}^{2}\right)^{3}} \\
& =-4 \frac{J}{\sqrt{\lambda}} z^{3}\left(1-\hat{g}^{2}\right) \int_{1}^{\infty} \frac{\mathrm{d} \omega}{\pi} \frac{\left(1-2 \hat{g}^{2}\right) \omega^{2}+\hat{g}^{2}}{\left(\omega^{2}-\hat{g}^{2}\right)^{3}} \\
& =\frac{J}{\sqrt{\lambda}}\left(z-\frac{1+2 z^{2}}{\sqrt{1+z^{2}}} \log \left[z+\sqrt{1+z^{2}}\right]\right)
\end{aligned}
$$

which exactly agrees with the expression (13) obtained in reference [19]. 


\subsection{The HL phase}

The non-analytic contribution is given by the the HL phase (22) through the coefficients $c_{r, s}^{(1)}$ of (26). More precisely,

$$
\operatorname{Non-analytic}(x)=\frac{1}{\pi} \frac{x^{2}}{x^{2}-g^{2} / 2} \int_{a}^{b} \mathrm{~d} y \rho_{0}(y)[\Delta \phi(x, y)+\Delta \phi(x,-y)],
$$

where

$$
\Delta \phi(x, y)=\frac{1}{2} \sum_{r=2}^{\infty} \sum_{m=0}^{\infty} c_{r, 2 m+r+1}^{(1)}\left(\frac{g}{\sqrt{2}}\right)^{2 m+2 r-1}\left(\frac{1}{x^{r} y^{2 m+r+1}}-\frac{1}{x^{2 m+r+1} y^{r}}\right) .
$$

Notice that we have taken into account the fact that the full set of Bethe roots is symmetrically distributed around zero by forming the combination $[\Delta \phi(x, y)+\Delta \phi(x,-y)]$. Let us define

$$
\omega=\frac{x}{a}, \quad \nu=\frac{y}{a},
$$

The double sum above can be carried out explicitly and gives

$\Delta \phi(\omega, \nu)=-\frac{1}{a^{2}}\left\{\frac{2 \hat{g}}{(\nu-\omega)\left(\nu \omega-\hat{g}^{2}\right)}+\left(\frac{1}{(\nu-\omega)^{2}}+\frac{\hat{g}^{2}}{\left(\nu \omega-\hat{g}^{2}\right)^{2}}\right) \log \left[\frac{(\omega-\hat{g})(\nu+\hat{g})}{(\omega+\hat{g})(\nu-\hat{g})}\right]\right\}$.

Furthermore,

$$
\begin{aligned}
\Delta \phi(\omega, \nu) & +\Delta \phi(\omega,-\nu)= \\
-\frac{4}{a^{2}} & \left\{\frac{\hat{g}\left(\hat{g}^{2}+\nu^{2}\right) \omega}{\left(\nu^{2}-\omega^{2}\right)\left(\nu^{2} \omega^{2}-\hat{g}^{4}\right)}+\omega \nu\left(\frac{1}{\left(\omega^{2}-\nu^{2}\right)^{2}}+\frac{\hat{g}^{4}}{\left(\nu^{2} \omega^{2}-\hat{g}^{4}\right)^{2}}\right) \log \left[\frac{\nu+\hat{g}}{\nu-\hat{g}}\right]\right. \\
& \left.+\frac{1}{2}\left(\frac{\omega^{2}+\nu^{2}}{\left(\omega^{2}-\nu^{2}\right)^{2}}+\frac{\hat{g}^{2}\left(\nu^{2} \omega^{2}+\hat{g}^{4}\right)}{\left(\nu^{2} \omega^{2}-\hat{g}^{4}\right)^{2}}\right) \log \left[\frac{\omega-\hat{g}}{\omega+\hat{g}}\right]\right\} .
\end{aligned}
$$

The correction to the energy (76) is then given by

$$
\left(E_{1}\right)_{\text {Bethe }}^{\text {non-analytic }}=-2 a \frac{k \hat{g}^{2}}{1-k \hat{g}^{2}} \oint_{\mathcal{C}^{+}} \frac{\mathrm{d} \omega}{2 \mathrm{i} \pi} \frac{\sqrt{\omega^{2}-1} \sqrt{\omega^{2}-\frac{1}{k^{2}}}}{\omega^{2}} \times \operatorname{Non-analytic}(\omega) \text {. }
$$

In the limit (4) and in the variables used here, the contour $\mathcal{C}^{+}$transforms into the real half line $[1,+\infty[$. The non-analytic part of the energy will therefore be given by the following double integral :

$$
\begin{aligned}
& \left(E_{1}\right)_{\text {Bethe }}^{\text {non-analytic }}= \\
& \quad \frac{4 n a^{2}}{\pi^{2}} \log (\alpha) \hat{g}^{2} \oint_{\mathcal{C}^{+}} \frac{\mathrm{d} \omega}{2 \mathrm{i} \pi} \frac{\sqrt{1-\omega^{2}}}{\omega^{2}-\hat{g}^{2}} \int_{1}^{\infty} \mathrm{d} \nu \frac{\nu \sqrt{\nu^{2}-1}}{\nu^{2}-\hat{g}^{2}}[\Delta \phi(\omega, \nu)+\Delta \phi(\omega,-\nu)] .
\end{aligned}
$$

This integration is carried out in the Appendix. The result reads

$$
\begin{aligned}
& \left(E_{1}\right)_{\text {Bethe }}^{\text {non-analytic }}=-\frac{n \log (\alpha)}{2 \pi \hat{g}}\left(2 \hat{g}^{2}+\left(3-\hat{g}^{2}\right) \log \left(1-\hat{g}^{2}\right)+\left(1+\hat{g}^{2}\right) \log \left(1+\hat{g}^{2}\right)\right) \\
& =\frac{J}{\sqrt{\lambda}} \frac{1}{\sqrt{1+z^{2}}}\left(-z^{2}+2\left(1+z^{2}\right) \log \left(1+z^{2}\right)-\left(1+2 z^{2}\right) \log \left(\sqrt{1+2 z^{2}}\right)\right)
\end{aligned}
$$

Here again, our result matches perfectly with the expression (15) from reference [19]. 


\section{Conclusion}

We have extracted the strong coupling limit of the scaling function for low twist, high spin operators of $\mathcal{N}=4 \mathrm{SYM}$ from the quantum string Bethe equations by applying these to a folded string rotating with angular momenta $(S, J)$ in $A d S_{3} \times S^{1} \subset A d S_{5} \times S^{5}$ and considering the limit

$$
1 \ll J \ll S, \quad z=\frac{\sqrt{\lambda}}{\pi J} \log \left(\frac{S}{J}\right), \quad \text { fixed } .
$$

It is interesting to notice that this limit which was observed in [18] and further explored in [19] from the string theory perspective also follows naturally from the quantum string Bethe ansatz. Namely, assuming the simplest possible analyticity structure with two cuts one is led to the relation (51) and using the rewritings in eqns. (61) to (63) the quantity $z$ naturally appears.

Our computation involved first a solution of the Bethe equations at the classical level. This part was straightforward and immediately led to the square root expression (65) for the classical energy. Subsequently, we determined the one-loop contribution to the energy. This contribution consisted of two parts, one originating from spin chain finite size effects and one being due to the Hernandez-Lopez phase. Both parts could be treated exactly and led to a total expression for the string one-loop energy, $\frac{J}{\sqrt{\lambda}} F(z)$, which agreed at the functional level with the result of a traditional string theory computation, cf. eqns. (13), (15), (179) and (87). Both the classical and the one loop energy when considered as a function of $z$ could be smoothly extrapolated to large values of $z$ and led to the strong coupling limit of the scaling function

$$
f(\lambda)=\frac{\sqrt{\lambda}}{\pi}-\frac{3 \log (2)}{\pi}+\mathcal{O}\left(\frac{1}{\sqrt{\lambda}}\right) .
$$

We stress again that the famous $\frac{-3 \log (2)}{\pi}$ is due to a highly non-trivial cancellation between terms originating from the HL-phase and terms due to spin chain finite size effects. More precisely, we have

$$
\begin{aligned}
E_{1}^{\text {analytic }} & \sim\left(\frac{-2 \log (z)+1-2 \log (2)}{\pi}\right) \log \left(\frac{S}{J}\right) \quad \text { as } \quad z \rightarrow \infty, \\
E_{1}^{\text {non-analytic }} & \sim\left(\frac{2 \log (z)-1-\log (2)}{\pi}\right) \log \left(\frac{S}{J}\right) \quad \text { as } \quad z \rightarrow \infty .
\end{aligned}
$$

As mentioned earlier there exists a numerical prediction for the coefficient of the $\mathcal{O}(1 / \sqrt{\lambda})$ term of (89) [16]. Furthermore, a genuine string theory calculation of the same quantity seems to be under way [30]. Given these developments it might be interesting to pursue our approach to two-loop order. It is obvious that the same strategy should be applicable and we are convinced that the Bethe equations will once again prove their efficiency.

Acknowledgments The authors thank Lisa Freyhult, Sergey Frolov and Matthias Staudacher for useful discussions. Both authors were supported by ENRAGE (European 
Network on Random Geometry), a Marie Curie Research Training Network financed by the European Community's Sixth Framework Programme, network contract MRTN-CT2004-005616.

\section{Appendix}

In this appendix, we present the evaluation of the double integral (86) which up to a factor $-\frac{16 n \hat{g}^{2}}{\pi^{2}} \log (\alpha)$ can be written as

$$
\mathcal{I}=\oint_{\mathcal{C}^{+}} \frac{\mathrm{d} \omega}{2 \mathrm{i} \pi} \frac{\sqrt{1-\omega^{2}}}{\omega^{2}-\hat{g}^{2}} \int_{1}^{\infty} \mathrm{d} \nu \frac{\nu \sqrt{\nu^{2}-1}}{\nu^{2}-\hat{g}^{2}}(\mathcal{A}(\omega, \nu)+\mathcal{B}(\omega, \nu))
$$

where

$$
\begin{aligned}
& \mathcal{A}(\omega, \nu)=\frac{\hat{g}\left(\hat{g}^{2}+\nu^{2}\right) \omega}{\left(\nu^{2}-\omega^{2}\right)\left(\nu^{2} \omega^{2}-\hat{g}^{4}\right)}+\omega \nu\left(\frac{1}{\left(\omega^{2}-\nu^{2}\right)^{2}}+\frac{\hat{g}^{4}}{\left(\nu^{2} \omega^{2}-\hat{g}^{4}\right)^{2}}\right) \log \left[\frac{\nu+\hat{g}}{\nu-\hat{g}}\right], \\
& \mathcal{B}(\omega, \nu)=\frac{1}{2}\left(\frac{\omega^{2}+\nu^{2}}{\left(\omega^{2}-\nu^{2}\right)^{2}}+\frac{\hat{g}^{2}\left(\nu^{2} \omega^{2}+\hat{g}^{4}\right)}{\left(\nu^{2} \omega^{2}-\hat{g}^{4}\right)^{2}}\right) \log \left[\frac{\omega-\hat{g}}{\omega+\hat{g}}\right],
\end{aligned}
$$

and the contour $\mathcal{C}^{+}$is the real half line $[1,+\infty$. The integration of $\mathcal{A}(\omega, \nu)$ with respect to $\omega$ is straightforward :

$$
\begin{aligned}
\oint_{\mathcal{C}^{+}} \frac{\mathrm{d} \omega}{2 \mathrm{i} \pi} \frac{\sqrt{1-\omega^{2}}}{\omega^{2}-\hat{g}^{2}} \mathcal{A}(\omega, \nu)= & -\operatorname{Res}_{\omega=\hat{g}}\left[\frac{\sqrt{1-\omega^{2}}}{\omega^{2}-\hat{g}^{2}} \mathcal{A}(\omega, \nu)\right]-\operatorname{Res}_{\omega=\frac{\hat{g}^{2}}{\nu}}\left[\frac{\sqrt{1-\omega^{2}}}{\omega^{2}-\hat{g}^{2}} \mathcal{A}(\omega, \nu)\right] \\
= & -\frac{\left(\hat{g}^{2}+\nu^{2}\right) \sqrt{1-\hat{g}^{2}}-\nu \sqrt{\nu^{2}-\hat{g}^{4}}}{2 \hat{g}\left(\hat{g}^{2}-\nu^{2}\right)^{2}} \\
& -\frac{\left(\hat{g}^{4}+\nu^{2} \hat{g}^{2}-2 \nu^{2}+4 \sqrt{1-\hat{g}^{2}} \nu \sqrt{\nu^{2}-\hat{g}^{4}}\right)}{4\left(\hat{g}^{2}-\nu^{2}\right)^{2} \sqrt{\nu^{2}-\hat{g}^{4}}} \log \left(\frac{\nu+\hat{g}}{\nu-\hat{g}}\right)
\end{aligned}
$$

In order to integrate the last term of (92), one first exploits the parity of the integrand to extend the contour $\mathcal{C}_{+}$to $\mathcal{C}_{+} \cup \mathcal{C}_{-}$(see Figure 1). Subsequently, the resulting contour can be deformed into the contour $\mathcal{C}_{0}$ around the cut $[-\hat{g}, \hat{g}]$. This contour integral can then be re-expressed as the finite part of the integral along $[-\hat{g}, \hat{g}]$ with the substitution

$$
\log \left[\frac{\omega-\hat{g}}{\omega+\hat{g}}\right] \longrightarrow 2 \mathrm{i} \pi
$$



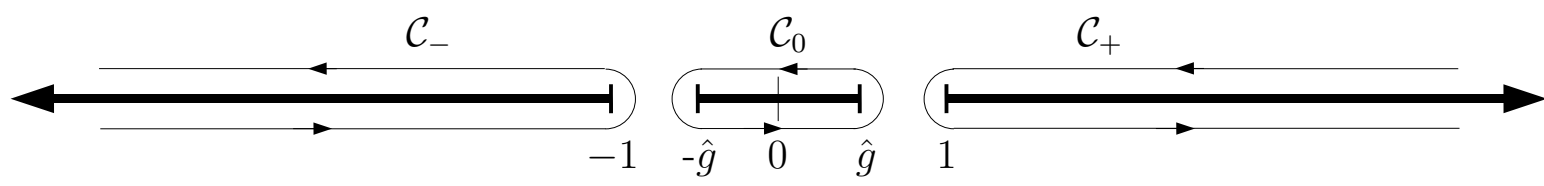

Figure 1: Cuts on the complex plane in the integration of $\mathcal{B}(\omega, \nu)$.

One gets

$$
\begin{aligned}
\oint_{\mathcal{C}^{+}} \frac{\mathrm{d} \omega}{2 \mathrm{i} \pi} \frac{\sqrt{1-\omega^{2}}}{\omega^{2}-\hat{g}^{2}} \mathcal{B}(\omega, \nu)= & -\frac{1}{2} \oint_{\mathcal{C}_{0}} \frac{\mathrm{d} \omega}{2 \mathrm{i} \pi} \frac{\sqrt{1-\omega^{2}}}{\omega^{2}-\hat{g}^{2}} \mathcal{B}(\omega, \nu) \\
= & \frac{1}{2} \int_{-\hat{g}}^{\hat{g}} \mathrm{~d} \omega \frac{\sqrt{1-\omega^{2}}}{\omega^{2}-\hat{g}^{2}} \frac{1}{2}\left(\frac{\omega^{2}+\nu^{2}}{\left(\omega^{2}-\nu^{2}\right)^{2}}+\frac{\hat{g}^{2}\left(\nu^{2} \omega^{2}+\hat{g}^{4}\right)}{\left(\nu^{2} \omega^{2}-\hat{g}^{4}\right)^{2}}\right) \\
= & \frac{\left(\hat{g}^{2}+\nu^{2}\right) \sqrt{1-\hat{g}^{2}}-\nu \sqrt{\nu^{2}-\hat{g}^{4}}}{2 \hat{g}\left(\hat{g}^{2}-\nu^{2}\right)^{2}} \\
& -\frac{\nu\left(\hat{g}^{2}+\nu^{2}-2\right)}{2\left(\nu^{2}-\hat{g}^{2}\right)^{2} \sqrt{\nu^{2}-1}} \tan ^{-1}\left(\frac{\hat{g} \sqrt{\nu^{2}-1}}{\nu \sqrt{1-\hat{g}^{2}}}\right) \\
& +\frac{\hat{g}^{4}+\left(\hat{g}^{2}-2\right) \nu^{2}}{2\left(\nu^{2}-\hat{g}^{2}\right)^{2} \sqrt{\nu^{2}-\hat{g}^{4}}} \tanh ^{-1}\left(\frac{\hat{g}^{3}}{\nu+\sqrt{1-\hat{g}^{2}} \sqrt{\nu^{2}-\hat{g}^{4}}}\right) \\
& -\frac{\sqrt{1-\hat{g}^{2}}\left(\hat{g}^{2}+\nu^{2}\right)}{2\left(\nu^{2}-\hat{g}^{2}\right)^{2} \hat{g}} \log \left(1-\hat{g}^{2}\right) .
\end{aligned}
$$

Here, $f_{-\hat{g}}^{\hat{g}}$ means that all poles in the interval $[-\hat{g}, \hat{g}]$ should be subtracted from the integrand. Summing (93) and (96), we obtain

$$
\begin{aligned}
\oint_{\mathcal{C}^{+}} \frac{\mathrm{d} \omega}{2 \mathrm{i} \pi} \frac{\sqrt{1-\omega^{2}}}{\omega^{2}-\hat{g}^{2}}(\mathcal{A}(\omega, \nu)+\mathcal{B}(\omega, \nu))= & -\frac{\sqrt{1-\hat{g}^{2}}\left(\hat{g}^{2}+\nu^{2}\right)}{2\left(\nu^{2}-\hat{g}^{2}\right)^{2} \hat{g}} \log \left(1-\hat{g}^{2}\right) \\
& -\frac{2 \sqrt{1-\hat{g}^{2}} \nu}{\left(\nu^{2}-\hat{g}^{2}\right)^{2}} \tanh ^{-1}\left(\frac{\hat{g}}{\nu}\right) \\
& -\frac{\nu\left(\hat{g}^{2}+\nu^{2}-2\right)}{2\left(\nu^{2}-\hat{g}^{2}\right)^{2} \sqrt{\nu^{2}-1}} \tan ^{-1}\left(\frac{\hat{g} \sqrt{\nu^{2}-1}}{\nu \sqrt{1-\hat{g}^{2}}}\right) \\
& -\frac{\hat{g}^{4}+\left(\hat{g}^{2}-2\right) \nu^{2}}{2\left(\nu^{2}-\hat{g}^{2}\right)^{2} \sqrt{\nu^{2}-\hat{g}^{4}}} \tanh ^{-1}\left(\frac{\hat{g} \sqrt{1-\hat{g}^{2}}}{\sqrt{\nu^{2}-\hat{g}^{4}}}\right) .
\end{aligned}
$$

The next task is to integrate (97) with respect to $\nu$. This can be done using the same kind of techniques as previously after rewriting the integral as a contour integral around $\mathcal{C}^{+}$. By this strategy, one gets the following intermediate results : 


$$
\begin{aligned}
& \int_{1}^{+\infty} \frac{\nu \sqrt{\nu^{2}-1}}{\nu^{2}-\hat{g}^{2}} \frac{\hat{g}^{2}+\nu^{2}}{\left(\nu^{2}-\hat{g}^{2}\right)^{2}} \mathrm{~d} \nu=\frac{\pi}{8} \frac{2-\hat{g}^{2}}{\left(1-\hat{g}^{2}\right)^{3 / 2}} \\
& \int_{1}^{+\infty} \frac{\nu \sqrt{\nu^{2}-1}}{\nu^{2}-\hat{g}^{2}} \frac{\nu}{\left(\hat{g}^{2}-\nu^{2}\right)^{2}} \tanh ^{-1}\left(\frac{\hat{g}}{\nu}\right) \mathrm{d} \nu \\
& =-\frac{\pi}{32 \hat{g}\left(1-\hat{g}^{2}\right)^{3 / 2}}\left(1-\frac{3}{2} \hat{g}^{2}+\frac{1}{\hat{g}^{2}} \log \left(1-\hat{g}^{2}\right)\right), \\
& \int_{1}^{+\infty} \frac{\nu \sqrt{\nu^{2}-1}}{\nu^{2}-\hat{g}^{2}} \frac{\nu\left(\hat{g}^{2}+\nu^{2}-2\right)}{\left(\hat{g}^{2}-\nu^{2}\right)^{2} \sqrt{\nu^{2}-1}} \tan ^{-1}\left(\frac{\hat{g}}{\nu} \frac{\sqrt{\nu^{2}-1}}{\sqrt{1-\hat{g}^{2}}}\right) \mathrm{d} \nu \\
& =-\frac{\pi}{16 \hat{g}\left(1-\hat{g}^{2}\right)}\left(1-\frac{3}{2} \hat{g}^{2}+\frac{1-\hat{g}^{4}}{\hat{g}^{2}} \log \left(1-\hat{g}^{2}\right)\right) \text {, } \\
& \int_{1}^{+\infty} \frac{\nu \sqrt{\nu^{2}-1}}{\nu^{2}-\hat{g}^{2}} \frac{\left(\hat{g}^{4}+\left(\hat{g}^{2}-2\right) \nu^{2}\right) \tanh ^{-1}\left(\frac{\hat{g} \sqrt{1-\hat{g}^{2}}}{\sqrt{\nu^{2}-\hat{g}^{4}}}\right)}{\left(\hat{g}^{2}-\nu^{2}\right)^{2} \sqrt{\nu^{2}-\hat{g}^{4}}} \mathrm{~d} \nu \\
& =\frac{\pi}{16 \hat{g}\left(1-\hat{g}^{2}\right)}\left(1-\frac{5}{2} \hat{g}^{2}-\frac{1-\hat{g}^{4}}{\hat{g}^{2}} \log \left(1+\hat{g}^{2}\right)\right) \text {. }
\end{aligned}
$$

Finally, putting everything together we end up with

$$
\mathcal{I}=\frac{\pi}{32 \hat{g}^{3}}\left(2 \hat{g}^{2}+\left(3-\hat{g}^{2}\right) \log \left(1-\hat{g}^{2}\right)+\left(1+\hat{g}^{2}\right) \log \left(1+\hat{g}^{2}\right)\right)
$$

which, up to the factor $-\frac{16 n \hat{g}^{2}}{\pi^{2}} \log (\alpha)$, gives back the result (87).

\section{References}

[1] J. A. Minahan and K. Zarembo, JHEP 0303, 013 (2003), hep-th/0212208; N. Beisert, C. Kristjansen and M. Staudacher, Nucl. Phys. B 664 (2003) 131, hep-th/0303060; N. Beisert and M. Staudacher, Nucl. Phys. B 670 (2003) 439, hep-th/0307042.

[2] G. Mandal, N. V. Suryanarayana and S. R. Wadia, Phys. Lett. B 543, 81 (2002), hep-th/0206103 I. Bena, J. Polchinski and R. Roiban, hep-th/0305116. JHEP 0310 (2003) 017, hep-th/0308089.

[3] J. M. Maldacena, Adv. Theor. Math. Phys. 2 (1998) 231 [Int. J. Theor. Phys. 38 (1999) 1113], hep-th/9711200.

[4] M. Staudacher, JHEP 0505, 054 (2005), hep-th/0412188. 
[5] N. Beisert, hep-th/0511082.

[6] N. Beisert, B. Eden and M. Staudacher, J. Stat. Mech. 0701, P021 (2007), hep-th/0610251

[7] N. Beisert, R. Hernandez and E. Lopez, JHEP 0611 (2006) 070, hep-th/0609044.

[8] Z. Bern, M. Czakon, L. J. Dixon, D. A. Kosower and V. A. Smirnov, Phys. Rev. D 75 (2007) 085010, hep-th/0610248; F. Cachazo, M. Spradlin and A. Volovich, Phys. Rev. D 75 (2007) 105011, hep-th/0612309.

[9] N. Beisert and M. Staudacher, Nucl. Phys. B 727, 1 (2005), hep-th/0504190.

[10] B. Eden and M. Staudacher, J. Stat. Mech. 0611, P014 (2006), hep-th/0603157.

[11] S. S. Gubser, I. R. Klebanov and A. M. Polyakov, Nucl. Phys. B 636 (2002) 99, hep-th/0204051.

[12] S. Frolov and A. A. Tseytlin, JHEP 0206, 007 (2002), hep-th/0204226.

[13] A. V. Kotikov and L. N. Lipatov, Nucl. Phys. B 769 (2007) 217, hep-th/0611204.

[14] L. F. Alday, G. Arutyunov, M. K. Benna, B. Eden and I. R. Klebanov, JHEP 0704 (2007) 082, hep-th/0702028.

[15] I. Kostov, D. Serban and D. Volin, hep-th/0703031.

[16] M. K. Benna, S. Benvenuti, I. R. Klebanov and A. Scardicchio, Phys. Rev. Lett. 98, 131603 (2007), hep-th/0611135.

[17] M. Beccaria, G. F. De Angelis and V. Forini, JHEP 0704 (2007) 066, hep-th/0703131.

[18] A. V. Belitsky, A. S. Gorsky and G. P. Korchemsky, Nucl. Phys. B 748 (2006) 24, hep-th/0601112.

[19] S. Frolov, A. Tirziu and A. A. Tseytlin, Nucl. Phys. B 766 (2007) 232, hep-th/0611269.

[20] G. Arutyunov, S. Frolov and M. Staudacher, JHEP 0410 (2004) 016 hep-th/0406256.

[21] R. Hernandez and E. Lopez, JHEP 0607, 004 (2006), hep-th/0603204; L. Freyhult and C. Kristjansen, Phys. Lett. B 638 (2006) 258, hep-th/0604069. N. Gromov and P. Vieira, hep-th/0703191.

[22] N. Beisert, A. A. Tseytlin and K. Zarembo, Nucl. Phys. B 715 (2005) 190, hep-th/0502173; R. Hernandez, E. Lopez, A. Perianez and G. Sierra, JHEP 0506 (2005) 011, hep-th/0502188.

[23] S. Frolov and A. A. Tseytlin, Nucl. Phys. B 668, 77 (2003), hep-th/0304255.

[24] N. Beisert, S. Frolov, M. Staudacher and A. A. Tseytlin, JHEP 0310, 037 (2003), hep-th/0308117. 
[25] N. Beisert and T. Klose, J. Stat. Mech. 0607 (2006) P006, hep-th/0510124.

[26] I. K. Kostov, Mod. Phys. Lett. A 4 (1989) 217.

[27] I. K. Kostov and M. Staudacher, Nucl. Phys. B 384 (1992) 459, hep-th/9203030.

[28] B. Eynard and C. Kristjansen, Nucl. Phys. B 455 (1995) 577, hep-th/9506193; Nucl. Phys. B 466, 463 (1996), hep-th/9512052.

[29] B. Basso and G. P. Korchemsky, hep-th/0612247.

[30] R. Roiban, A. Tirziu and A. A. Tseytlin, arXiv:0704.3638 [hep-th]. 